\title{
Laparoscopic view of endosalpingiosis in a woman with dermoid cyst and endometriosis
}

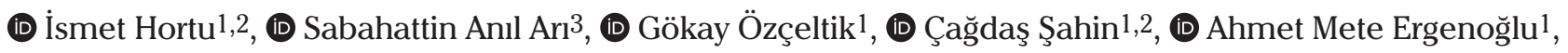 \\ (D) Fuat Akercan ${ }^{1}$ \\ 1Department of Obstetrics and Gynecology, Ege University Faculty of Medicine, İzmir, Turkey \\ 2Department of Stem Cell, Ege University Institute of Health Sciences, İzmir, Turkey \\ ${ }^{3}$ Clinic of Obstetrics and Gynecology, Menemen State Hospital, İzmir, Turkey
}

\section{Abstract}

\begin{abstract}
Endosalpingiosis is, like endometriosis, the presence of cystic masses outside of the salpinx which contains fallopian tube epithelium. Endosalpingiosis can be seen on the surface of ovaries, tubal serosa, uterine serosa, myometrium, and also in the bladder. The main clinical features of endosalpingiosis are pelvic pain, adnexal mass which mimics cancer, and urinary symptoms. Herein, we present a surgical video of endosalpingiosis in a woman with endometriosis and a dermoid cyst.
\end{abstract}

Keywords: Endosalpingiosis, laparoscopy, endometriosis, dermoid cyst

Received: 06 April, 2020 Accepted: 20 July, 2020

\section{Introduction}

Endosalpingiosis is, like endometriosis, the presence of cystic masses outside of the salpinx, which contains fallopian tube epithelium with affected structures that may include the ovarian cortex, uterine serosa, and the surface of other pelvic organs, and the inguinal region (15). Endosalpingiosis is usually an incidental finding at the time of surgery. Although endosalpingiosis is a benign and rare condition, it can mimic peritoneal cancer or metastases (6). Experienced pathologists are crucial for exact diagnosis. Endosalpingiosis differs histologically from endometriosis since it has ciliated glandular epithelium, no endometriumlike tissue, and does not display the same inflammatory reactions. Endosalpingiotic glands should be discriminated from mesonephric remnants in the pelvis, which are common incidental microscopic findings in the region of the fallopian tube. Mesonephric remnants are typically located more deeply than endosalpingiosis and characteristically have a collar of smooth muscle under the epithelial lining, which is typically a single layer of non-ciliated, low columnar to cuboidal cells. As in the present case, the endosalpingiotic tissue contains columnar and ciliated epithelium with intercalated cells, which possess a clear cytoplasm $(7,8)$. Thus it is important to raise awareness of endosalpingiosis, but radical surgery should be limited, due to high recurrence rates.

The purpose of this video article (Video 1) was to demonstrate a laparoscopic view of incidental endosalpingiosis, concomitant with dermoid cyst and endometriosis. This operation was recorded at a university hospital. A 40-yearold woman was admitted to our outpatient clinic due to pelvic pain with a duration of six months. Her medical history included cesarean section and laparoscopic ovarian cyst surgery. Transvaginal ultrasonography revealed a 5 $\mathrm{cm}$ dermoid cyst in the right adnexal area. Tumor markers and other biochemical parameters were within the normal range. In light of the findings, laparoscopic surgery was proposed. A $10 \mathrm{~mm}$ trocar was inserted through the umbilicus for the optic system and three ancillary ports were also employed. Endoscopic visualization revealed the right 
ovary with a $5 \mathrm{~cm}$ cyst, diffuse clear cystic masses involving the uterine serosa and an endometriotic lesion on the vesico-uterine peritoneal fold (Figure 1,2). Left ovary and other organs were of normal appearence. The cyst wall was cauterized with a bipolar instrument. During dissection via laparoscopic scissors, the cyst was punctured. Cyst content was aspirated, immediately. Then, the dermoid cyst wall was extirpated with a traction counter-traction technique. The cyst wall was placed in a surgical sterile surgical glove and removed via a $10 \mathrm{~mm}$ optic port. The pelvic peritoneal cavity was thoroughly washed with sterile saline. Small bleeds were cauterized with the bipolar instrument and then the right ovary was sutured. A punch biopsy was taken from the clear cysts on the uterus. After the coagulation of the endometriotic lesions on the pelvis, the operation was terminated (Figure 3). Histopathological diagnosis of the punch biopsy material from the clear cysts on the uterus was reported as endosalpingiosis.

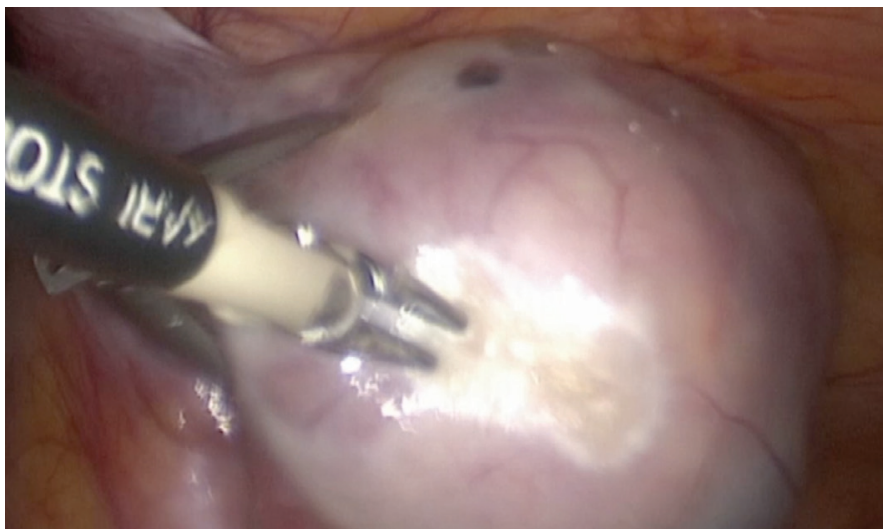

Figure $1.5 \mathrm{~cm}$ diameter dermoid cyst in the right ovary

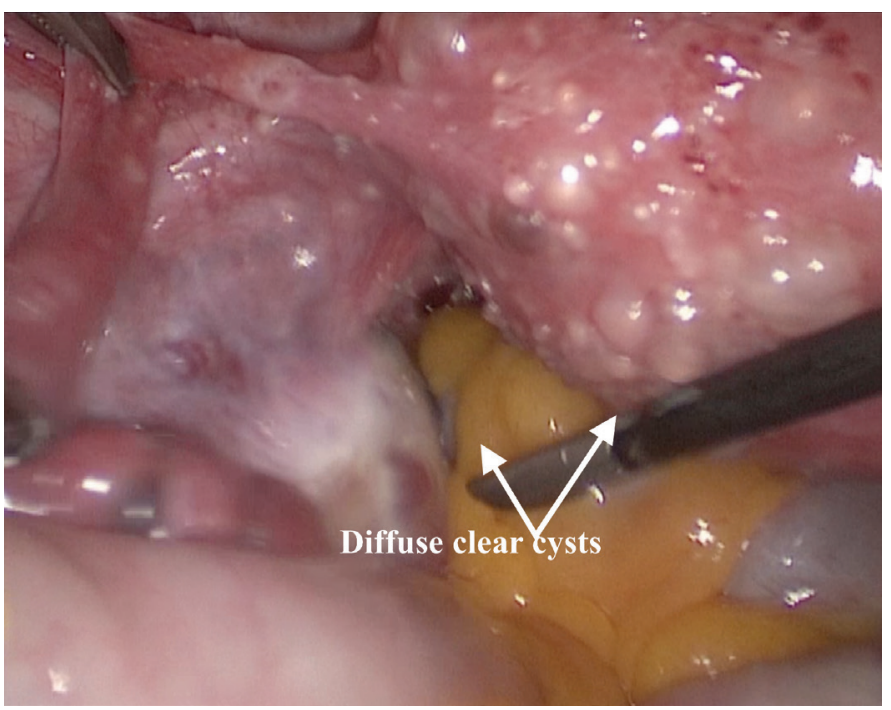

Figure 2. Diffuse clear cysts on the surface of the uterus, fallopian tube, and the left ovary

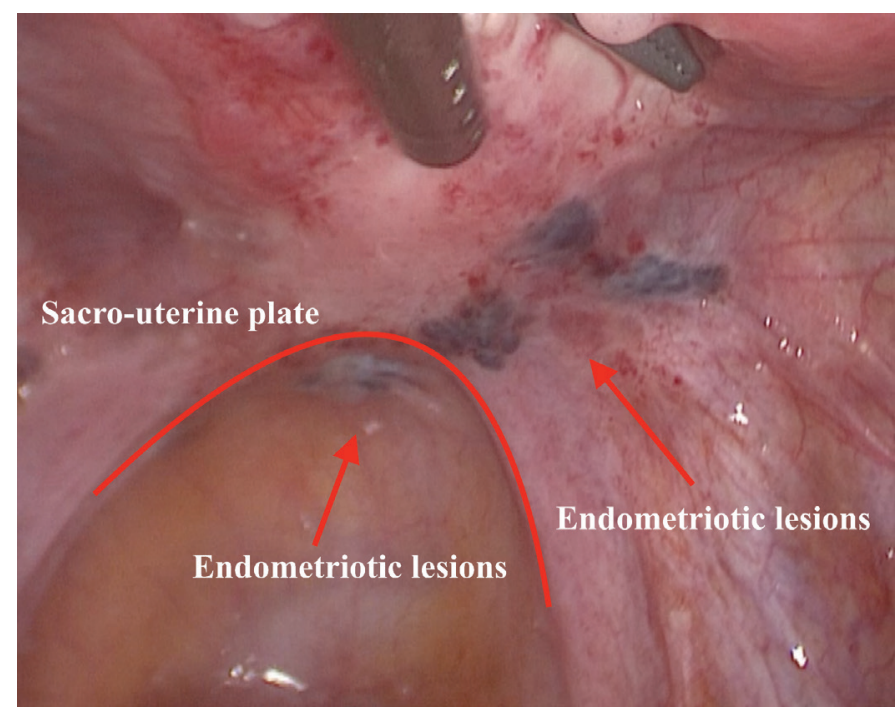

Figure 3. Endometriotic lesions on the peritoneum of the pouch of Douglas

Video 1. Stepwise demonstration of the operation with narrated video footage

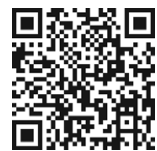

https://www.doi.org/10.4274/jtgga.galenos.2020.2020.0052.video1

Informed Consent: Informed consent was obtained from the patient.

Conflict of Interest: No conflict of interest was declared by the authors.

Financial Disclosure: The authors declared that this study received no financial support.

\section{References}

1. Prentice L, Stewart A, Mohiuddin S, Johnson NP. What is endosalpingiosis? Fertil Steril 2012; 98: 942-7.

2. Batt RE, Yeh J. Müllerianosis: four developmental (embryonic) mullerian diseases. Reprod Sci 2013; 20: 1030-7.

3. Laganà AS, Vitale SG, Salmeri FM, Triolo O, Frangež HB, VrtačnikBokal, et al. Unus pro omnibus, omnes pro uno: A novel, evidencebased, unifying theory for the pathogenesis of endometriosis. Med Hypotheses 2017; 103: 10-20.

4. Laganà AS, Garzon S, Götte M, Viganò P, Franchi M, Ghezzi F, et al. The pathogenesis of endometriosis: molecular and cell biology insights. Int J Mol Sci 2019; 20: 5615.

5. Stojanovic M, Brasanac D, Stojicic M. Cutaneous inguinal scar endosalpingiosis and endometriosis: case report with review of literature. Am J Dermatopathol 2013; 35: 254-60. 
6. Rajarubendra N, Leang Y, Monsour M. Mullerianosis of the urinary bladder. ANZ J Surg 2015; 85: 292-3.

7. McCoubrey A, Houghton O, McCallion K, McCluggage WG. Serous adenocarcinoma of the sigmoid mesentery arising in cystic endosalpingiosis. J Clin Pathol 2005; 58: 1221-3.
8. Carrick KS, Milvenan JS, Albores-Saavedra J. Serous tumor of low malignant potential arising in inguinal endosalpingiosis: report of a case. Int J Gynecol Pathol 2003; 22: 412-5. 